\title{
Front Matter: Volume 8829
}

, "Front Matter: Volume 8829," Proc. SPIE 8829, Organic Light Emitting Materials and Devices XVII, 882901 (17 October 2013); doi:

$10.1117 / 12.2045836$

SPIE Event: SPIE Organic Photonics + Electronics, 2013, San Diego, California, SPIE. United States 


\title{
PROCEEDINGS OF SPIE
}

\section{Organic Light Emitting Materials and Devices XVII}

\author{
Franky So \\ Chihaya Adachi \\ Editors
}

\section{5-28 August 2013}

San Diego, California, United States

Sponsored by

SPIE

Cosponsored by

Aldrich Materials Science (United States)

EMD Millipore Corporation (d.b.a. EMD Chemicals) (United States)

Published by

SPIE 
The papers included in this volume were part of the technical conference cited on the cover and title page. Papers were selected and subject to review by the editors and conference program committee. Some conference presentations may not be available for publication. The papers published in these proceedings reflect the work and thoughts of the authors and are published herein as submitted. The publisher is not responsible for the validity of the information or for any outcomes resulting from reliance thereon.

Please use the following format to cite material from this book:

Author(s), "Title of Paper," in Organic Light Emitting Materials and Devices XVII, edited by

Franky So, Chihaya Adachi, Proceedings of SPIE Vol. 8829 (SPIE, Bellingham, WA, 2013) Article CID Number.

ISSN: 0277-786X

ISBN: 9780819496799

Published by

SPIE

P.O. Box 10, Bellingham, Washington 98227-0010 USA

Telephone +1 3606763290 (Pacific Time) · Fax +1 3606471445

SPIE.org

Copyright (@ 2013, Society of Photo-Optical Instrumentation Engineers.

Copying of material in this book for internal or personal use, or for the internal or personal use of specific clients, beyond the fair use provisions granted by the U.S. Copyright Law is authorized by SPIE subject to payment of copying fees. The Transactional Reporting Service base fee for this volume is $\$ 18.00$ per article (or portion thereof), which should be paid directly to the Copyright Clearance Center (CCC), 222 Rosewood Drive, Danvers, MA 01923. Payment may also be made electronically through CCC Online at copyright.com. Other copying for republication, resale, advertising or promotion, or any form of systematic or multiple reproduction of any material in this book is prohibited except with permission in writing from the publisher. The CCC fee code is 0277-786X/13/\$18.00.

Printed in the United States of America.

Publication of record for individual papers is online in the SPIE Digital Library.

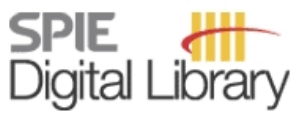

SPIEDigitalLibrary.org

Paper Numbering: Proceedings of SPIE follow an e-First publication model, with papers published first online and then in print and on CD-ROM. Papers are published as they are submitted and meet publication criteria. A unique, consistent, permanent citation identifier (CID) number is assigned to each article at the time of the first publication. Utilization of CIDs allows articles to be fully citable as soon as they are published online, and connects the same identifier to all online, print, and electronic versions of the publication. SPIE uses a six-digit CID article numbering system in which:

- The first four digits correspond to the SPIE volume number.

- The last two digits indicate publication order within the volume using a Base 36 numbering

system employing both numerals and letters. These two-number sets start with 00, 01, 02, 03, 04, $05,06,07,08,09,0 A, 0 B \ldots$. 0Z, followed by 10-1Z, 20-2Z, etc.

The CID Number appears on each page of the manuscript. The complete citation is used on the first page, and an abbreviated version on subsequent pages. Numbers in the index correspond to the last two digits of the six-digit CID Number. 


\section{Contents}

vii Conference Committee

MATERIALS

882906 Novel host materials for blue phosphorescent OLEDs [8829-5]

P. Strohriegl, D. Wagner, P. Schrögel, S. T. Hoffmann, A. Köhler, Univ. Bayreuth (Germany);

U. Heinemeyer, I. Münster, BASF SE (Germany)

882907 Novel oligonuclear copper complexes featuring exciting luminescent characteristics (Invited Paper) [8829-6]

D. M. Zink, cynora GmbH (Germany); D. Volz, L. Bergmann, cynora GmbH (Germany) and Karlsruhe Institute of Technology (Germany); M. Nieger, Univ. of Helsinki (Finland); S. Bräse, Karlsruhe Institute of Technology (Germany); H. Yersin, Univ. Regensburg (Germany);

T. Baumann, cynora GmbH (Germany)

882909 White organic light emitting diodes using Pt-based red, green, and blue phosphorescent dopants (Invited Paper) [8829-8]

B. O'Brien, Arizona State Univ. (United States) and Flexible Display Ctr. (United States); G. Li, T. Fleetham, J. Li, Arizona State Univ. (United States)

8829 OA Conjugated polymer particles: towards self-assembling organic photonics (Invited Paper) [8829-9]

A. J. C. Kuehne, T. Luelf, M. Wessling, RWTH Aachen e.V. (Germany); J. Sprakel,

Wageningen Univ. (Netherlands)

\section{DEVICE PHYSICS I}

$8829 \mathrm{OH}$ Performance improvement of fluorescent blue organic emitting diode by insertion buffer layer between hole-transporting layer and emitting layer [8829-16]

B. Lee, J. Park, D. Lee, D. Kim, J. Park, Duksan Hi-Metal Co., Ltd. (United States)

$8829 \mathrm{ON}$ Analysis of hole mobility of fluorene derivative films based on the disorder model and relationship between disorder free mobility and reorganization energy in the Marcus theory [8829-22]

M. Era, Saga Univ. (Japan); K. Mori, Ryouka Systems Inc. (Japan); N. Mototsu, Advanced Technology Research Labs. (Japan) 
8829 OR Novel technologies for commercialized 55-inch WRGB OLED TV (Invited Paper) [8829-26] Y.-H. Tak, C.-W. Han, H.-S. Kim, B.-C. Kim, J.-W. Kim, T.-S. Kim, B.-S. Kim, C.-H. Oh, S.-Y. Cha, B.-C. Ahn, LG Display (Korea, Republic of)

8829 OS Reliable six PEP LTPS device for AMOLED's (Invited Paper) [8829-27] C.-W. Chou, P.-Y. Wang, C.-W. Hu, Y. Chang, C.-S. Chuang, Y. Lin, AU Optronics Corp. (Taiwan)

\section{SOLUTION PROCESSABLE SMALL MOLECULAR OLEDS I}

8829 OV Solution processable small molecular host materials for blue and white phosphorescence OLEDs (Invited Paper) [8829-30]

Y.-T. Lee, National Taiwan Univ. (Taiwan) and Academia Sinica (Taiwan); Y.-T. Chang,

C.-T. Chen, Academia Sinica (Taiwan); C.-T. Chen, National Taiwan Univ. (Taiwan)

$8829 \mathrm{OZ}$ All solution processed blue multi-layer light emitting diodes realized by thermal layer stabilization and orthogonal solvent processing [8829-34]

S. Nau, R. Trattnig, NanoTecCenter Weiz Forschungsgesellschaft (Austria); L. Pevzner, MaxPlanck-Institut für Polymerforschung (Germany); M. Jäger, NanoTecCenter Weiz Forschungsgesellschaft mbH (Germany); R. Schlesinger, M. V. Nardi, G. Ligorio,

C. Christodoulou, Humbold-Univ. zu Berlin (Germany); N. Schulte, Merck KGaA (Germany); S. Winkler, Helmholz Zentrum Berlin für Materialien und Energie GmbH (Germany); J. Frisch, Humbold-Univ. zu Berlin (Germany); A. Vollmer, Helmholz Zentrum Berlin für Materialien und Energie GmbH (Germany); M. Baumgarten, Max-Planck-Institut für Polymerforschung (Germany); S. Sax, NanoTecCenter Weiz Forschungsgesellschaft mbH (Austria); N. Koch, Humboldt-Univ. zu Berlin (Germany) and Helmholz Zentrum Berlin für Materialien und Energie GmbH (Germany); K. Müllen, Max-Planck-Institut für Polymerforschung (Germany); E. J. W. List-Kratochvil List, Graz Univ.of Technology (Austria) and NanoTecCtr. Weiz Forschungsgesellschaft mbH (Austria)

\section{NOVEL DEVICES AND LASERS}

882918 Polymer nanofibers as novel light-emitting sources and lasing material (Invited Paper) [8829-43]

A. Camposeo, L. Persano, National Nanotechnology Lab. of Istituto Nanoscienze, CNR (Italy) and Ctr. for Biomolecular Nanotechnologies, Istituto Italiano di Tecnologia (Italy); D. Pisignano, National Nanotechnology Lab. of Istituto Nanoscienze, CNR (Italy), Ctr. for Biomolecular Nanotechnologies, Istituto Italiano di Tecnologia (Italy), and Univ. del Salento (Italy)

882919 Recent progress on the vacuum deposition of OLEDs with feature sizes $\leq \mathbf{2 0} \boldsymbol{\mu m}$ using a contact shadow mask patterned in-situ by laser ablation [8829-44] Y. Kajiyama, K. Joseph, Univ. of Waterloo (Canada); K. Kajiyama, S. Kudo, V-Technology Co., Ltd. (Japan); H. Aziz, Univ. of Waterloo (Canada) 
SSL-OLEDS

8829 1B Candle light-style OLED: a plausibly human-friendly safe night light (Invited Paper) [8829-45]

J.-H. Jou, C.-Y. Hsieh, P.-Y. Chen, National Tsing Hua Univ. (Taiwan); C.-C. Wang,

C.-C. Chen, F.-C. Tung, S.-H. Chen, Y.-S. Wang, Industrial Technology Research Institute

(Taiwan)

8829 1C Carrier-gas enhanced vapor phase deposition for organic thin films: addressing mass manufacturing requirements for OLED devices, and overcoming existing challenges with OVPD (Invited Paper) [8829-46]

J. Kreis, M. Schwambera, D. Keiper, M. Gersdorff, M. Long, M. Heuken, AIXTRON SE (Germany)

8829 lE Flexible top-emitting OLEDs for lighting: bending limits [8829-48]

P. Schwamb, T. Reusch, Osram Opto Semiconductors (Germany); C. J. Brabec, FriedrichAlexander-Univ. of Erlangen-Nuremberg (Germany) and Bavarian Ctr. of Applied Energy Research (Germany)

\section{LIGHT EXTRACTION}

8829 1H Optical design for efficient light emission in OLEDs and anisotropic layers (Invited Paper) [8829-51]

L. Penninck, K. Neyłs, Ghent Univ. (Belgium)

882911 Improved light outcoupling and mode analysis of top-emitting OLEDs on periodically corrugated substrates [8829-52]

T. Schwab, C. Fuchs, R. Scholz, Technische Univ. Dresden (Germany); X. Li, F. Xie, W. Choy, The Univ. of Hong Kong (Hong Kong, China); K. Leo, M. C. Gather, Technische Univ.

Dresden (Germany)

8829 IL Device reflectivity as a simple rule for predicting the suitability of scattering foils for improved OLED light extraction [8829-55]

J. W. Levell, S. Harkema, R. K. Pendyala, P. A. Rensing, A. Senes, TNO (Netherlands);

D. Bollen, Agfa-Gevaert (Belgium); D. MacKerron, DuPont Teijin Films U.K. (United Kingdom);

J. S. Wilson, TNO (Netherlands)

8829 1M Enhanced light out-coupling from surface plasmonic loss minimized transparent organic light-emitting diodes [8829-56]

J.-B. Kim, J.-H. Lee, C.-K. Moon, S.-Y. Kim, J.-J. Kim, Seoul National Univ. (Korea, Republic of)

POSTER SESSION

8829 IR New platinum complexes for hybrid white organic light-emitting diodes [8829-61]

A. Poloek, National Taiwan Univ. (Taiwan) and Academia Sinica (Taiwan); C.-T. Chen,

Academia Sinica (Taiwan); C.-T. Chen, National Taiwan Univ. (Taiwan) 
8829 IU Synthesis of fluorene-based polyelectrolytes tethering different counterions for singlecomponent white light-emitting electrochemical cells [8829-64]

S.-H. Yang, C.-S. Tsai, B.-C. Liu, H.-C. Su, National Chiao Tung Univ. (Taiwan)

$88291 \mathrm{~V}$ The far-field optical distribution profiles and properties of a planar white organic lightemitting diode [8829-65]

H. Yang, Y.-C. Lian, L.-J. Chen, W.-S. Li, National Taipei Univ. of Technology (Taiwan)

8829 IW Improvement of operation voltage and efficiency in inverted blue phosphorescent organic light-emitting devices [8829-66]

C.-H. Chang, H.-S. Huang, Y.-D. SU, Y.-H. Liang, Y.-S. Chang, C.-H. Chiu, Yuan Ze Univ.

(Taiwan); H.-H. Chang, Vanung Univ. (Taiwan)

8829 IX Recent progress on solution processable small molecules for organic light-emitting diodes in ITRI [8829-67]

T.-C. Chao, J.-Y. Liao, H.-C. Yeh, J.-S. Lin, M.-R. Tseng, Industrial Technology Research Institute (Taiwan)

882920 Correlation between exciton-induced degradation of organic/metal interfaces and energy barrier for electron injection at organic/metal interfaces in organic optoelectronic devices [8829-70]

Q. Wang, H. Aziz, Univ. of Waterloo (Canada)

882923 OLED emission zone measurement with high accuracy [8829-73]

N. Danz, R. MacCiarnain, D. Michaelis, Fraunhofer-IOF (Germany); T. Wehlus, A. F. Rausch, OSRAM Opto Semiconductors (Germany); C. A. Wächter, Fraunhofer-IOF (Germany);

T. C. G. Reusch, OSRAM Opto Semiconductors (Germany)

882926 High-throughput quantum chemistry and virtual screening for OLED material components [8829-77]

M. D. Halls, D. J. Giesen, T. F. Hughes, A. Goldberg, Y. Cao, Schrödinger Inc. (United States)

882927 Low roll-off and high efficiency orange OLEDs using green and red dopants in an exciplex forming co-host [8829-78]

S. Lee, K.-H. Kim, S.-J. Yoo, Y.-S. Park, J.-J. Kim, Seoul National Univ. (Korea, Republic of)

882929 Correlation between shift in recombination zone and efficiency roll-off in phosphorescent organic light emitting devices (PHOLEDs) [8829-81]

H. Zamani Siboni, H. Aziz, Univ. of Waterloo (Canada)

\section{SOLUTION PROCESSABLE SMALL MOLECULAR OLEDS III}

8829 2B Admittance analysis in (PPE-PPV) polymer (AnE-PVstat) light emitting diodes [8830-92]

O. Özdemir, Yildiz Technical Unv. (Turkey); S. P. Mucur, TUBITAK (Turkey) and Gebze Institute of Technology (Turkey); E. Tekin, TUBITAK (Turkey); S. Boudiba, Linz Institude for Organic Solar Cells (Austria) and Univ. de Tébessa (Algeria); C. Ulbricht, Johannes Kepler Univ.

(Netherlands); D. A. M. Egbe, Linz Institude for Organic Solar Cells (Austria);

U. Dereb Menda, P. Kavak, K. Kutlu, Yildiz Technical Unv. (Turkey)

Author Index

vi 


\title{
Conference Committee
}

\author{
Symposium Chair
}

Zakya H. Kafafi, National Science Foundation (United States)

Conference Chair

Franky So, University of Florida (United States)

Conference CoChair

Chihaya Adachi, Kyushu University (Japan)

Conference Program Committee

Malte C. Gather, Technische Universität Dresden (Germany)

Hisao Ishii, Chiba University (Japan)

Hironori Kaji, Kyoto University (Japan)

Jang-Joo Kim, Seoul National University (Korea, Republic of)

Jaewon Lee, LG Display (Korea, Republic of)

Mathew K. Mathai, Plextronics, Inc. (United States)

Jongwook Park, The Catholic University of Korea (Korea, Republic of)

Yong-Jin Pu, Yamagata University (Japan)

Ifor D. W. Samuel, University of St. Andrews (United Kingdom)

Joseph Shinar, lowa State University (United States)

Richard J. Wilson, Cambridge Display Technology Ltd. (United Kingdom)

\section{Session Chairs}

1 Materials

Chihaya Adachi, Kyushu University (Japan)

Jang-Joo Kim, Seoul National University (Korea, Republic of)

2 Device Physics I

Ifor D. W. Samuel, University of St. Andrews (United Kingdom)

Russell J. Holmes, University of Minnesota, Twin Cities (United States)

3 Device Physics II

Joseph Shinar, lowa State University (United States)

Bernard Kippelen, Georgia Institute of Technology (United States)

4 Solution Processable Small Molecular OLEDs I

Joseph Shinar, lowa State University (United States)

Bernard Kippelen, Georgia Institute of Technology (United States) 
5 Solution Processable Small Molecular OLEDs II

Malte C. Gather, Technische Universität Dresden (Germany)

Daisuke Yokoyama, Yamagata University (Japan)

6 Novel Devices and Lasers

Malte C. Gather, Technische Universität Dresden (Germany)

Daisuke Yokoyama, Yamagata University (Japan)

$7 \quad$ SSL-OLEDS

Denis Kondakov, DuPont (United States)

John C. de Mello, Imperial College London (United Kingdom)

8 Light Extraction

Denis Kondakov, DuPont (United States)

John C. de Mello, Imperial College London (United Kingdom) 